\title{
ON THE CLASSIFICATION OF MAGNETIC CATACLYSMIC VARIABLES
}

\author{
Paul A. Mason ${ }^{1}$ \\ RESUMEN
}

Se presenta un esquema para clasificar las Variables Cataclísmicas Magnéticas (MCVs). Cuando las MCVs descubiertas eran diez o veinte fue sencillo clasificarlas basándose en sus propiedades observacionales directas. Ahora que el número de MCVs excede el centenar, el método para clasificarlas necesita ser actualizado. Una consideración importante es la posibilidad de que las binarias puedan cambiar físicamente su clasificación, por ejemplo ¿llegarán polares intermedias a convertirse en polares conforme evolucionan hacia períodos orbitales más cortos? $\mathrm{O}$, ¿podrá transformarse una polar en una polar intermedia al romperse el sincronismo debido a ina nova?

\section{ABSTRACT}

The classification scheme for Magnetic Cataclysmic Variables (MCVs) is addressed. When only ten or twenty MCVs had been discovered their classification was simple and based on direct observational properties. Now that the number of MCVs exceeds one hundred, the method of classification needs updating. One important consideration is the possibility that binaries might physically change their classification. For example will intermediate polars become polars as they evolve to shorter orbital periods? Or does a polar become an intermediate polar when synchronism is broken due to a nova?

\section{Key Words: BINARIES: CLOSE - NOVAE, CATACLYSMIC VARIABLES - STARS: MAGNETIC}

\section{INTRODUCTION}

The beginning of the field of magnetic cataclysmic variable stars (MCVs)can be marked with Tapia's (1976) discovery of strongly modulated optical polarization from AM Herculis, along with subsequent observations of AM Her, AN UMa, EF Eri, and VV Pup, established the canonical description of a polar. That is a cataclysmic variable star (i.e. a white dwarf accreting from a lower main sequence secondary) with x-ray and optical light curves, circular polarization and optical/UV emission lines all modulating with the same period. Along with a physical interpretation involving disk-less accretion and magnetic locking of the binary with the rotation of the white dwarf. The current magnetic field range of white dwarfs in polars is $10-230 \mathrm{MG}$.

Another group of objects included the prototype DQ Her and were thought to be MCVs with fields less than the polars. These binaries were thought to have accretion disks that were truncated at which point the accretion flow was magnetically channelled to the white dwarf's surface. For some time these asynchronous MCVs were divided, by some authors (e.g. Warner 1992) into two classes based on the ratio of their white dwarf spin and their binary orbital periods. The intermediate polars (IPs) tended

\footnotetext{
${ }^{1}$ The University of Texas at El Paso, Department of Physics, El Paso, TX, 79968, USA
}

to have ratios near 0.1 while the DQ Herculis binaries (DQ Hers) had much lower ratios and it was inferred that the DQ Hers had weaker magnetic fields. Patterson (1994) referred to all of these objects as DQ Herculis stars and suggested that the presence of multiple periodicities be their defining characteristic.

\section{BLURRING THE CLASSIFICATIONS}

Given the open question of whether the IPs and DQ Hers should be considered as distinct sub-classes and no argument for a distinction between the AM Hers and the polars when there were less than 20 binaries in each class, the classification scheme seemed to be on solid ground. The discovery of new binaries soon changed things. The discovery of circular polarization from the IP BG CMi (West, Berriman, and Schmidt 1987) shook things up only slightly. Until then all polars were polarized (hence the name) and all IPs and/or DQ Hers were unpolarized. But given the fact that derived magnetic field for BG CMi (2-5 $M G$ ) was less than any polar, the separation of polars and IPs became based less on the presence of polarization and more on the synchronism of polars and the presence of disks in IPs.

\subsection{Asynchronous Polars and a Stream-fed IP}

The discovery of complex circular polarization curves of BY Cam (Mason, Liebert, and Schmidt 
1989 ) and the discovery that V1500 Cyg (Nova Cygni 1975) was a MCV (Schmidt, Stockman, and Lamb 1988) clearly blurred the distinction between polars and IPs. BY Cam showed all of the classic polar signatures; strong polarization, optical and $\mathrm{x}$-ray light curves and strong optical emission lines that modulate at (or at least approximately at) the same period and no indication of a disk. Extensive campaigns eventually concluded that the white dwarf in BY Cam rotates slightly faster than the binary orbit (Mason et al. 1995, Silber et al. 1997, Mason et al. 1998). V1500 Cyg appears to be a polar that was knocked out of synchronism by a nova in 1975. To complicate matters it seems that V1500 Cyg has (or at least had) a post-nova disk (Schmidt and Stockman 1991). Patterson (1994) suggested that these binaries should be called BY Cam stars, however the term asynchronous polars has stuck. In addition, the IP V2400 Oph has been described as a diskless or stream-fed IP (Buckley, et al. 1997), with a spin period that is 15.45 minutes and an orbital period of 205.8 minutes. So we have polars that defy the synchronism condition and even possibly the disk-less (or stream-fed) condition and an IP that apparently rotates very asynchronously without the formation of a disk. So what classification criterion can we count on?

\subsection{The Synchronization Parameter}

The synchronization parameter $\mathrm{S}$ (Mason and Gray, 2003) is defined by

$$
S=N_{m a g} / N_{a c c}
$$

where $N_{m a g}$ and $N_{a c c}$ are the magnetic and accretion torques respectively. If $S<1$ then the system is not synchronized. If $S>1$ then the white dwarf's rotation period will be synchronized (or will become synchronized if currently not in equilibrium) with the binary. The parameter $\mathrm{S}$ depends on the accretion rate, $\dot{M}$, in $g s^{-1}$, the white dwarf mass, $M_{w d}$, in solar units, the binary mass ratio, $\mathrm{q}$, the orbital period, $P_{\text {orb }}$, in hours, and the primary's magnetic field strength, $B_{\text {dip }}$, in MG, and $\gamma$ is the pitch angle of the connecting field lines. Following Warner (1996) equation (36),

$S=0.4 \times 10^{-17} \gamma \dot{M}^{-1} P_{\text {orb }}^{-1 / 12}(1+q)^{6 / 7} q^{0.213} M_{w d}^{1 / 2} B_{\text {dip }}$.

Where the mass radius relationship for the white dwarf,

$$
R=0.73 \times 10^{9} M_{w d}^{-1 / 3}
$$

(Warner 1996) is used, where $R$ is measured in $\mathrm{cm}$ and $M_{w d}$ is measured in solar masses.

\section{CONCLUSIONS}

The classification of polars with the sub-class of asynchronous polars makes sense if we consider polars as those systems which either are synchronized or will become synchronized once equilibrium is reached. That is those binaries with a synchronization parameter that is greater than one should be considered to be polars. NCVs with synchronization parameters that are less than one will not become polars unless conditions change enough to raise the synchronization parameter above unity.

The parameter $\mathrm{S}$ is most strongly dependent on $\dot{M}, \mathrm{q}$ and $B_{d i p}$. Considering the fact that the synchronization parameter depends only very weakly on orbital period, and hence orbital separation, it is unlikely that many of the known IPs will ever be polars based on the shortening of the binary separation alone. A significant reduction in accretion rate must also occur. Andrew Norton (2004) discussed the nearly synchronous IPs at this conference. On the other hand it, is possible that marginally locked polars exist. That is a polar with an $\mathrm{S}$ parameter that is near unity and may drop below unity as the result of periods of high accretion rate and above unity during low states.

I am grateful to Andrew Norton for a discussion of work he presented at this conference, Koji Mukai for a discussion on several specific systems, and for the help of my student Candace Gray.

\section{REFERENCES}

Buckley, D. A. H., Haberl, F., Motch, C., Pollard, K., Schwarzenberg-Czerny, A., and Sekiguchi, K., 1997. MNRAS, 287, 117

Mason, P. A. and Gray, C. 2003, in "Magnetic Cataclysmic Variables", ASP Conf. Series, in press

Mason, P. A., Liebert, J. and Schmidt, G. D. 1989, ApJ, 346, 941

Mason, P. A., Andronov, I. L., Kolesnikov, S. V.. Pavlenko, E. P., and Shakovskoy, N. M. 1995, ASP Conf. Ser., 85, 496

Mason, P. A., Ramsay, G., Andronov, I., Kolesnikov, S., Shakhovskoy, N. and Pavlenko, E., 1998, MNRAS. 295,511

Norton, A. 2004, these proceedings

Patterson, J. 1994, PASP, 106, 209

Silber et al. 1997, MNRAS, 290, 25

Tapia, S. 1976, IAUC 2987

Schmidt, G. D. and Stockman, H. S. 1991, ApJ, 371, 719

Schmidt, G. D., Stockman, H. S., and Lamb, D. Q. $198 \mathrm{~S}$ ApJ, 332, 282

Warner, B. 1993, in ASP Conf. Ser., 29, 2.42

Warner, B. 1996, Ast. Sp. Sci., 241, 263

West, S. C., Berriman, G., and Schmidt, G. D. 1987. ApJ. 322, L35 